\title{
Affine line systems in finite-dimensional vector spaces
}

\author{
Rainer Löwen, Günter F. Steinke and Hendrik Van Maldeghem* \\ Dedicated to Professor Adriano Barlotti on the occasion of his 80 th birthday
}

\begin{abstract}
A topological translation plane may be defined as a system $\mathscr{L}$ of affine $l$-dimensional subspaces of $\mathbb{R}^{2 l}$ such that (1) any two points are joined by a unique line $L \in \mathscr{L}$ and joining is continuous; (2) Euclid's parallel postulate holds and $\mathscr{L}$ is invariant under translation. It is easy to see that one of the two parts of condition (2) may be omitted. We use topological methods to show that (2) may be omitted altogether, unless $l=8$. In other words, we show that, except possibly for $l=8$, there are no proper stable planes with point set $\mathbb{R}^{2 l}$ and affine subspaces as lines, and that translation invariance is automatic. The core of the argument is Theorem 3.1, which characterizes the lines of a topological translation plane among affine subspaces: an $l$-dimensional subspace $A$ is a line if there is a point $p \notin A$ such that every line passing through $p$ intersects $A$ in at most one point. We also consider the effect of the dual spread condition in our context. Moreover, we point out limits to generalization by giving examples obtained by transfinite induction in finite dimensional vector spaces over arbitrary infinite skew fields.
\end{abstract}

Key words. Topological translation plane, compact spread, linear space.

2000 Mathematics Subject Classification. Primary: 51H10; Secondary: 51A05, 51A40

\section{Linear planes}

1.1 Definition. a) A join space $(V, \mathscr{L})$ consists of a set $V$ of points and a system $\mathscr{L}$ of subsets $L \subseteq V$, called lines, such that any two points $x, y \in V$ are joined by a unique line $L=x \vee y \in \mathscr{L}$.

b) We call $(V, \mathscr{L})$ a linear plane if, in addition to the above, the following conditions hold:

(LP 1) $V$ is a vector space over a skew field $F$,

(LP 2) all lines are affine subspaces of $V$, and

(LP 3) any two intersecting lines are cosets of complementary vector subspaces.

* Parts of this paper have been intensely discussed with several colleagues. The authors wish to thank T. Grundhöfer, H. Hähl, L. Kramer, H. Löwe, B. Polster, R. Riesinger, N. Rosehr, M. Stroppel. 
More precisely, condition (LP 3 ) says that for any two lines $K, L \in \mathscr{L}$ intersecting in a point $x=K \wedge L$ we have a vector space decomposition $V=(K-x) \oplus(L-x)$.

In the literature, join spaces are usually called linear spaces. We want the term 'linear' to indicate the presence of a vector space structure. Condition (LP 3) justifies the name 'plane' given to these structures.

Observe that the case $\operatorname{dim}_{F} V=2$ is trivial: in this case, every one-dimensional subspace must occur as a line, hence $(V, \mathscr{L})$ is the Desarguesian affine plane over $F$.

1.2 Proposition. For a linear plane, the following conditions are equivalent:

(TP 1) Euclid's parallel postulate holds in $(V, \mathscr{L})$, that is, $(V, \mathscr{L})$ is an affine plane,

(TP 2) The line system is invariant under vector space translation, that is, $\mathscr{L}+V=\mathscr{L}$.

Proof. 1) Suppose that condition (TP 2) holds. Given $L \in \mathscr{L}$ and $x \in V \backslash L$, choose $y \in L$; then the line $K=L+x-y$ contains $x$ and is disjoint from $L$. We show that every other line $K^{\prime}$ containing $x$ intersects $L$. Indeed, the vector subspaces $K-x$ and $K^{\prime}-x$ are complementary, hence there are $k \in K$ and $k^{\prime} \in K^{\prime}$ such that $k-x+$ $k^{\prime}-x=y-x$, and then $y-(k-x)=k^{\prime} \in L \cap K^{\prime}$.

2) Conversely, suppose that $(V, \mathscr{L})$ is an affine plane and consider $L \in \mathscr{L}$ and $v \in V$ such that $L+v \neq L$. We have to show that $L+v \in \mathscr{L}$. Pick $y \in L$; then $x=y+v \notin L$ and there is a line $K$ line that contains $x$ and is parallel to $L$.

We claim that $L+v \subseteq K$. If there is $z \in L$ such that $z+v \notin K$, then the line $W=x \vee(z+v)$ intersects $L$, say $L \wedge W=w$. Then $L+v=L+x-w$, and the subspace $W \cap L=W \cap(L+v)-x+w$ is at least 1-dimensional. This implies that $L=W$, a contradiction to $x \notin L$.

Equality $K=L+v$ now follows by applying twice what we have shown. Indeed, $K-v$ is contained in the line $M$ passing through $y$ parallel to $K$, hence $L \subseteq$ $K-v \subseteq M$. Since both $L$ and $M$ are lines, the three sets are equal.

1.3 Translation planes. Affine planes which are linear planes (and hence satisfy (TP 2)) are called affine translation planes. For a group theoretical definition of affine translation planes, see [1]; more background and countless examples can be found in the books [4], [13], and [17].

Observe that a translation plane $(V, \mathscr{L})$ is completely determined by the pencil $\mathscr{L}_{0}$ of all lines containing the origin 0 ; indeed, we have $\mathscr{L}=\mathscr{L}_{0}+V$. The pencil is a partition, that is, a covering of $V$ by mutually complementary vector subspaces. This carries over to linear planes in general:

1.4 Proposition. Let $(V, \mathscr{L})$ be a linear plane. Then for each $v \in V$, the pencil $\mathscr{L}_{v}$ of all lines containing $v$ defines a partition $\mathscr{L}_{v}-v$ of $V$ into mutually complementary vector subspaces. In particular, $\left(V, \mathscr{L}_{v}+V\right)$ is a translation plane.

If the translation planes defined by distinct points are all identical, then the given linear plane is itself a translation plane. In the finite case, there are no linear planes other than translation planes: 


\subsection{Proposition. Every finite linear plane is an affine translation plane.}

Proof. It follows from (LP 3) that all lines are of the same finite dimension over $F$. Hence, they all contain the same number $n$ of points, and $V$ has $n^{2}$ points. The number of lines passing through the origin is therefore $\frac{n^{2}-1}{n-1}=n+1$. A line $L$ not passing through 0 meets exactly $n$ of these lines, hence it has a unique parallel. The assertion follows in view of 1.2.

\subsection{Problem 1. Are there any linear planes that are not affine translation planes?}

At the end of the present section, we shall give a positive answer to this question using transfinite induction. Examples of this kind are not very pleasant, hence we shall sharpen the problem by adding continuity conditions:

1.7 Problem $1^{\prime}$. Are there any weakly topological linear planes that are not affine translation planes?

The definition of 'weakly topological' will be given in Section 2, and in Section 3 we shall give a partial, negative answer to Problem $1^{\prime}$. The following observation should help to develop some feeling for the problems.

In an affine translation plane, all lines parallel to a given one form a set of mutually disjoint affine subspaces, all of the same dimension, covering the whole vector space $V$. In the case of translation planes, any set of lines with these properties is necessarily translation invariant. One might wonder if this is true for affine subspaces in general, and if this could lead to a negative answer to Problem 1. However, it is rather easy to give examples that are not translation invariant. The examples given below are even continuous in the sense that the subspace containing a point $p$ depends on $p$ continuously, hence we do not even obtain negative evidence with respect to Problem 1'.

1.8 Example. The vector space $\mathbb{R}^{4}$ can be covered by a set of mutually disjoint 2dimensional affine subspaces that is not translation invariant. We obtain such an example using a $(2 \times 2)$-matrix $A$ having no real eigenvalue, for instance $A=\left(\begin{array}{cc}0 & -1 \\ 1 & 0\end{array}\right)$. For $x, y \in \mathbb{R}$, we set $(x, y) A=(u(x, y), v(x, y))$ and define

$$
W(x, y)=(x, y, 0,0)+\langle(0,0,1,0),(u(x, y), v(x, y), 0,1)\rangle,
$$

where the acute brackets denote the linear span. The condition for a vector $(a, b, s, t)$ to belong to $W(x, y)$ is that $(a, b)=(x, y)(\mathbb{0}+t A)$, where $\mathbb{1}$ denotes the $2 \times 2$ identity matrix. Hence there is a unique solution $(x, y)$. The affine spaces $W(x, y)$ for $x, y \in \mathbb{R}$ then form a partition of $\mathbb{R}^{4}$.

Observe that the horizontal subspaces

$$
W^{\prime}(u, v)=(0,0, u, v)+\langle(1,0,0,0),(0,1,0,0)\rangle
$$

for $u, v \in \mathbb{R}$ also form a partition of $\mathbb{R}^{4}$ and that furthermore any two subspaces 
$W(x, y)$ and $W^{\prime}(u, v)$ are complementary. However there is no 2-dimensional affine subspace that is complementary to each of $W(x, y)$ and $W^{\prime}(u, v)$.

For everything that follows, we need to fix our notation and terminology regarding affine and projective spaces.

1.9 Affine and projective spaces. Consider a (skew) field $F$ and a (left) $F$-vector space $W$ of finite dimension $n+1$. The $n$-dimensional projective space $\mathscr{P}(W)=\mathscr{P}_{n} F$ is the lattice of all vector subspaces of $W$. We denote by $P_{k}(W)$ the set of all subspaces $Y$ of (vector space) dimension $k+1$; they are considered as $k$-dimensional 'flats' or, briefly, $k$-flats in $\mathscr{P}(W)$, and we write $k=\operatorname{pdim} Y$ for the projective dimension. Given $X \in \mathscr{P}(W)$, we denote the set of all $k$-flats contained in $X$ by $P_{k}(X)$. Often we identify $X$ with its point set $P_{0}(X)$.

From an $n$-dimensional (left) $F$-vector space $V$, we obtain the $n$-dimensional affine space $\mathscr{A}(V)=\mathscr{A}_{n} F$; it consists of the point set $V=A_{0}(V)$ together with the set of all affine subspaces. By definition, an affine subspace is a coset $B=U+v, v \in V$, of a vector subspace $U \leqslant V$. In this situation, $U$ will be called the direction space of $B$ and denoted $U=B_{0}$. The affine span $0 \vee B=B_{0}+F v$ (the smallest affine subspace containing 0 and $B$ ) will be denoted $\tilde{B}$. The set of $k$-dimensional affine subspaces will be denoted $A_{k}(V)$. One-dimensional affine subspaces will be called $F$-lines in order to distinguish them from the lines of a linear plane.

Using $V=F^{n}$ and $W=F^{n+1}$, we obtain an embedding of $\mathscr{A}_{n} F$ into $\mathscr{P}_{n} F$ as follows. The subspace $H=F^{n} \times\{0\} \in P_{n-1}(W)$ is a hyperplane, and the complement $P_{0}(W) \backslash P_{0}(H)$ may be identified with $F^{n}$ via $\left(a_{1}, \ldots, a_{n}\right) \leftrightarrow F\left(a_{1}, \ldots, a_{n}, 1\right)$. Under this identification, every $k$-dimensional affine subspace $B \subseteq F^{n}$ corresponds to a set of the form $P_{0}(X) \backslash P_{0}(H)$, where $X \in P_{k}(W)$ is uniquely determined, and we write $X=\bar{B}$. We call $H$ the hyperplane at infinity with respect to this embedding of $\mathscr{A}_{n} F$ into of $\mathscr{P}_{n} F$. Thinking of $\bar{B}, B$ and $H$ as subsets of the point set $P_{0}(W)$, we form the complement $\bar{B} \backslash B=\bar{B} \cap H$ and denote it by $B_{\infty}$. It can be described as the set of all directions of $F$-lines contained in $B$.

We proceed now to the construction of linear planes that are not translation planes. The following lemma and its corollary seem to be well known, compare [18], but we provide proofs for the convenience of the reader.

1.10 Lemma. Let $V$ be a vector space of finite dimension over a skew field $F$. If $\mathscr{W}$ is a family of proper subspaces of cardinality card $\mathscr{W}<\operatorname{card} F$, then the union of $\mathscr{W}$ cannot be all of $V$.

Proof. Suppose that $V=\bigcup \mathscr{W}$, where all $W \in \mathscr{W}$ are proper vector subspaces of $V$. Clearly, $\mathscr{W}$ contains at least two elements of $W_{1}, W_{2}$ that are maximal with respect to inclusion. By induction on the dimension of $V$, we find elements $v_{1} \in$ $W_{1} \backslash \bigcup\left(\mathscr{W} \backslash\left\{W_{1}\right\}\right)$ and $v_{2} \in W_{2} \backslash \bigcup\left(\mathscr{W} \backslash\left\{W_{2}\right\}\right)$. Consider the vectors $w_{t}=v_{1}+t v_{2}$, where $t \in F$. For $t \neq t^{\prime}$, the vectors $w_{t}$ and $w_{t^{\prime}}$ must belong to different subspaces $W, W^{\prime} \in \mathscr{W}$, which proves that $\operatorname{card} F \leqslant \operatorname{card} \mathscr{W}$. Indeed, if both vectors belong to the same subspace $W \in \mathscr{W}$, then $w_{t}-w_{t^{\prime}}=\left(t-t^{\prime}\right) v_{2} \in W$ so that $v_{2} \in W$. Thus $W=W_{2}$ and $v_{1}=w_{t}-t v_{2} \in W_{2}-$ a contradiction to the choice of $v_{1}$. 
$\mathrm{N}$. Rosehr pointed out that some finiteness condition is indispensable for 1.10. For example, any vector space of countable dimension can be obtained as the union of a countable ascending chain of proper subspaces.

\subsection{Corollary. Let $V$ be an $n$-dimensional vector space over a skew field $F$ and let $\mathscr{W}$} be a family of vector subspaces of $V$ of dimension at most $m$, where $m<n$. If $\operatorname{card} \mathscr{W}<\operatorname{card} F$, then there is a vector subspace $U$ of $V$ of dimension $n-m$ such that $U \cap W=\{0\}$ for all $W \in \mathscr{W}$.

Proof. For each $W \in \mathscr{W}$, let $W^{(0)}=W$. If we have vector subspaces $W^{(k)}$ of dimension at most $m+k<n$, there is a vector $v_{k+1} \in V$ not contained in any of the $W^{(k)}$ by Lemma 1.10. We then define $W^{(k+1)}$ to be the linear span of $W^{(k)}$ and $v_{k+1}$. This process gives us $n-m$ vectors $v_{1}, v_{2}, \ldots, v_{n-m}$ such that the vector subspace $U$ spanned by them has dimension $n-m$. Furthermore, $U \cap W=\{0\}$ for all $W \in \mathscr{W}$.

1.12 Examples of non-affine linear planes. Consider an infinite skew field $F$ and a left $F$-vector space $V$ of finite dimension $2 l \geqslant 4$. Using transfinite induction, we shall construct a set $\mathscr{L}$ of $l$-dimensional affine subspaces of $V$ such that $V$ endowed with $\mathscr{L}$ as a line set becomes a linear plane.

Let $\omega$ be the smallest ordinal having the same cardinality as $F$. Then we know that for any $v \in \omega$, the set of all $\mu<v$ has smaller cardinality than $F$. Consider the following two sets, which have the same cardinality as $F$ : the set $\mathscr{R}$ of all $F$-lines (onedimensional affine subspaces) of $V$ and the set $\mathscr{U}$ of all $l$-dimensional affine subspaces of $V$. Choose bijections $v \mapsto R_{v}$ and $v \mapsto U_{v}$ of $\omega$ onto these sets. For each $\alpha \in \omega$, we shall define an $l$-dimensional subspace $L_{\alpha}$, and $\mathscr{L}$ will be the set of all these subspaces.

We shall enforce that we obtain a join space simply by making sure that every $R_{v} \in \mathscr{R}$ is contained in a unique line $L_{\alpha} \in \mathscr{L}$. Let $1 \in \omega$ be the smallest element. The only condition for the choice of $L_{1}$ is that $R_{1} \leqslant L_{1}$. If lines $L_{\beta}$ have been chosen for all $\beta<\alpha \in \omega$ such that (LP 3) is satisfied, consider the smallest $v=v(\alpha)$ such that $R_{v}$ is not contained in any of the $L_{\beta}, \beta<\alpha$. (To see that such $R_{v}$ exist, apply 1.11 to the direction spaces of the $L_{\beta}$.) We shall choose $L_{\alpha}$ such that it contains $R_{v(\alpha)}$; the problem is to ensure that (LP 3) is satisfied. In the sequel, we shall prove the existence of suitable candidates $U \in \mathscr{U}$ for $L_{\alpha}$. The transfinite enumeration of all $l$-dimensional subspaces will then be used to remove all ambiguity from the definition of $L_{\alpha}$ : among the spaces $U_{\mu}$ satisfying our conditions, we choose the one with minimal $\mu$. Our procedure guarantees that always $v(\alpha) \geqslant \alpha$, so that every element of $\mathscr{R}$ will eventually be contained in some line.

We may assume that $R_{v}$ contains the origin 0 . We define $V_{\beta}=\left(L_{\beta}\right)_{0}+R_{v}$ if the direction space $\left(L_{\beta}\right)_{0}$ does not contain $R_{v}$ and $V_{\beta}=\tilde{L}_{\beta}$ (the linear span) if $\left(L_{\beta}\right)_{0}$ contains $R_{v}$. In any case $V_{\beta}$ has dimension $l+1<2 l$. By Corollary 1.11 there is an $l-1$ dimensional vector subspace $W$ of $V$ that is complementary to each $V_{\beta}$. Let $U=W+R_{v}$. Then $\operatorname{dim} U=l$ and $U \cap V_{\beta}=R_{v}$. It now follows that $\left(L_{\beta}\right)_{0} \cap U=\{0\}$ if $\left(L_{\beta}\right)_{0}$ does not contain $R_{v}$ and $\tilde{L}_{\beta} \cap U=R_{v}$ if $\left(L_{\beta}\right)_{0}$ contains $R_{v}$.

Hence, if none of the direction spaces $\left(L_{\beta}\right)_{0}, \beta<\alpha$, contains $R_{v}$, then we may choose $L_{\alpha}$ complementary to all of them, which entails that $\operatorname{card}\left(L_{\alpha} \cap L_{\beta}\right)=1$. If 
some of the $\left(L_{\beta}\right)_{0}$ contain $R_{v}$, we can make our choice such that $\tilde{L}_{\beta} \cap L_{\alpha}=R_{v}$ for all such $\beta$. This ensures that $L_{\beta}$ and $L_{\alpha}$ are disjoint, but their direction spaces are different. This case inevitably occurs at some stage, hence we cannot get a translation plane. Actually, we do not get a single pair of lines that are translates of each other.

\section{Weakly topological linear planes}

Throughout this section, we consider real linear planes $(V, L)$ of finite dimension. In other words, $F=\mathbb{R}$ will be the field of real numbers, and the vector space $V=\mathbb{R}^{2 l}$ will be of finite dimension, necessarily an even number $2 l$. Then lines are $l$ dimensional affine subspaces. We shall assume that the join operation is continuous at least in one variable, and we shall deduce stronger continuity properties. In particular, it will follow that $l \in\{1,2,4,8\}$ (but, as we remarked in 1.1 , only the cases $l>1$ are interesting). The purpose of these results is not to initiate a theory of topological linear planes but to minimize the hypotheses of the non-existence result that will be proved in Section 3.

The vector space $V$ will be regarded as the real affine space $\mathscr{A}\left(\mathbb{R}^{2 l}\right)$ embedded in the real projective $2 l$-space $\mathscr{P}\left(\mathbb{R}^{2 l+1}\right)$, as explained in 1.9. We shall make use of the continuity properties of the geometric operations in real projective spaces, as explained in the sequel.

2.1 Real topological projective spaces. We abbreviate $\mathscr{P}=\mathscr{P}\left(\mathbb{R}^{n+1}\right)$ and $P_{k}=$ $P_{k}\left(\mathbb{R}^{n+1}\right)$, compare 1.9. Each of the sets $P_{k}$ carries a natural topology, the Grassmann topology, which makes it a compact topological (even smooth) manifold. The topology can either be derived from the transitive action of the general linear group GL $(W)$ on $P_{k}$, compare [19], 64.3, or from local coordinate systems, compare [5], VI, §3.5. The point set $P_{0}=P_{0}\left(\mathbb{R}^{n+1}\right)$ is the $n$-manifold $\mathbb{P}_{n}$, the real projective $n$ space. Its topology is simply the quotient topology with respect to the map $\mathbb{R}^{n+1} \backslash\{0\} \rightarrow P_{0}$ sending a vector $v \neq 0$ to its span $\mathbb{R} v$. The point set $P_{0}(X)$ of each $k$-flat $X \in P_{k}$ is homeomorphic to $\mathbb{P}_{k}$.

With respect to the Grassmann topologies, $\mathscr{P}$ is a topological projective space. This means, among other things, that the join and intersection operations $\vee$ and $\wedge$ are both continuous on each subset of $P_{k} \times P_{r}$ on which the result of the operation has constant dimension. Moreover, the function $\operatorname{pdim}(X \wedge Y)$ is upper semi-continuous on $P_{k} \times P_{r}$, which means that $\operatorname{pdim}\left(X^{\prime} \wedge Y^{\prime}\right) \leqslant \operatorname{pdim}(X \wedge Y)$ for all $\left(X^{\prime}, Y^{\prime}\right)$ in some small neighbourhood of $(X, Y)$. This will be referred to as the stability property. There is also a dual stability property for joins. Finally, we need the fact that the incident pairs form a closed subset of $P_{k} \times P_{r}$ for each $k \leqslant r$. A convenient reference for these results due to Misfeld is [14].

2.2 Topological translation planes. As we observed in 1.3, a translation plane $\left(\mathbb{R}^{2 l}, \mathscr{L}\right)$ with point set $\mathbb{R}^{2 l}$ is determined by the pencil $\mathscr{L}_{0}$ of all lines containing the origin 0 . The pencil is a partition, that is, a covering of $\mathbb{R}^{2 l}$ by mutually complementary $l$-dimensional vector subspaces.

If we think of $\mathbb{R}^{2 l}$ as real affine space embedded in real projective space $\mathscr{P}\left(\mathbb{R}^{2 l+1}\right)$, 
then we may view the pencil as a subset $\mathscr{L}_{0} \subseteq P_{l}\left(\mathbb{R}^{2 l+1}\right)$, from where it inherits a topology, referred to as the Grassmann topology. Taking intersections with the hyperplane $H$ at infinity, we obtain a spread $\mathscr{S} \subseteq P_{l-1}(H)$, that is, a set of mutually disjoint $(l-1)$-flats covering $H$. We have $P_{l-1}(H) \approx P_{l-1}\left(\mathbb{R}^{2 l}\right)$, and the topology induced on $\mathscr{S}$ by this set corresponds to the Grassmann topology because the projection map $\mathscr{L}_{0} \rightarrow \mathscr{S}$ and its inverse are both continuous.

Using $\mathscr{P}\left(\mathbb{R}^{2 l+1}\right)$, one obtains a neat description of the projective closure of $\left(\mathbb{R}^{2 l}, \mathscr{L}\right)$. Its point set is $\left(P_{0} \backslash H\right) \cup \mathscr{S}$, and its lines are the $l$-flats $L$ such that $L \wedge H$ belongs to the spread $\mathscr{S}$, plus one line represented by $\mathscr{S}$ itself. Incidence is given by inclusion. This description is known as the André-Bose representation of a projective translation plane; it can also be used in order to obtain the topology of the projective point set as a quotient topology inherited from $P_{0}\left(\mathbb{R}^{2 l+1}\right)$, see [15] or [19], 64.4. The topology of the line space is taken from $P_{l}\left(\mathbb{R}^{2 l+1}\right)$. It turns out that a spread $\mathscr{S}$ defines a topological translation plane (with continuous join and intersection) if and only if $\mathscr{S}$ is compact in the Grassmann topology. In this case, $\mathscr{S}$ is homeomorphic to the $l$-sphere $\mathbb{S}_{l}$, and $l \in\{1,2,4,8\}$, compare [19], 52.5.

We are now ready to consider continuity properties of linear planes. The following result sharpens the characterization of topological translation planes that we just mentioned; the additional fact is that the Grassmann topology on the line pencil of a translation plane is the only one that can render the join operation continuous even in one variable.

2.3 Proposition. Let $\left(\mathbb{R}^{2 l}, \mathscr{L}\right)$ be an affine translation plane and assume that the pencil $\mathscr{L}_{0}$ carries a Hausdorff topology $\tau$ such that the join map $j_{0}: \mathbb{R}^{2 l} \backslash\{0\} \rightarrow \mathscr{L}_{0}$, defined by $j_{0}(x)=x \vee 0$, is continuous. Then $\tau$ coincides with the Grassmann topology $\gamma$, and $\left(\mathbb{R}^{2 l}, \mathscr{L}\right)$ is a topological translation plane; in particular, $l \in\{1,2,4,8\}$.

Proof. 1) Let $\mathbb{S} \subseteq \mathbb{R}^{2 l}$ be a sphere centered at 0 . We consider a third topology on $\mathscr{L}_{0}$, namely, the quotient topology $\delta$ defined by the restriction $j_{0}: \mathbb{S} \rightarrow \mathscr{L}_{0}$. It is compact, and by assumption the identity map $\left(\mathscr{L}_{0}, \delta\right) \rightarrow\left(\mathscr{L}_{0}, \tau\right)$ is continuous. Since $\tau$ is assumed to be Hausdorff, the topologies agree.

2) We shall show that $\mathscr{L}_{0}$ is closed in $P_{l-1}\left(\mathbb{R}^{2 l}\right)$ with respect to the Grassmann topology and, hence, compact. This will imply that the join map is continuous with respect to $\gamma$, see 2.2. Repeating the argument of step (1) we obtain that $\gamma=\delta=\tau$. Everything else follows, see again 2.2.

Suppose therefore that a sequence of lines $L_{n} \in \mathscr{L}_{0}$ converges to an $l$-dimensional vector space $A \in P_{l-1}\left(\mathbb{R}^{2 l}\right) \backslash \mathscr{L}_{0}$ with respect to the Grassmann topology. Then $A \cap \mathbb{S}$ contains two points $a_{1}, a_{2}$ such that the lines $a_{1} \vee 0$ and $a_{2} \vee 0$ are distinct. Using the properties of the topological projective space $\mathscr{P}_{2 l} \mathbb{R}$, one obtains sequences $x_{n}^{i} \in$ $L_{n} \cap \mathbb{S}$ such that $x_{n}^{i} \rightarrow a_{i}$ for $i=1,2$. This implies that $L_{n}=j_{0}\left(x_{n}^{i}\right) \rightarrow a_{i} \vee 0$ with respect to $\tau$, but a sequence in a Hausdorff space cannot have two limits.

2.4 Definition. We say that a linear plane $\left(\mathbb{R}^{2 l}, \mathscr{L}\right)$ is weakly topological if there is a Hausdorff topology $\tau$ on $\mathscr{L}$ such that the join operation $\vee$ is continuous in each variable separately. For instance, if $\left(\mathbb{R}^{2 l}, \mathscr{L}\right)$ is a stable plane (see, e.g., [10]), then it 
is weakly topological. In fact, one can show like in 2.3 that continuity of join in both variables simultaneously implies that the linear plane is a stable plane with respect to the Grassmann topology. We do not give a proof, because at present we only have nonexistence results and no examples of topological proper linear planes.

From 1.4 and 2.3 we conclude that each line pencil $\mathscr{L}_{v}, v \in \mathbb{R}^{2 l}$, of a weakly topological linear plane defines a topological translation plane. In particular, we have $l \in\{1,2,4,8\}$ by 2.2 , and, since we are looking for linear planes that are not translation planes, we may assume that $l>1$.

2.5 The projective join map. Let $\left(\mathbb{R}^{2 l}, \mathscr{L}\right)$ be a topological translation plane. Consider an l-dimensional affine subspace $A \subseteq \mathbb{R}^{2 l}$ not containing the origin 0 . In addition, we assume that $A$ meets each line passing through 0 in at most one point. For example, $A$ could be a line of a linear plane having the pencil $\mathscr{L}_{0}$ in common with the given translation plane. We have the restriction $j=j_{A, 0}: A \rightarrow \mathscr{L}_{0}$ of the continuous join map. We want to extend this map to a continuous surjection $J=J_{A, 0}: \bar{A} \rightarrow \mathscr{L}_{0}$, where $\bar{A}$ is the projective closure of $A$ as defined in 1.9. (As usual, we are identifying $\bar{A}$ with its set of points $P_{0}(\bar{A}) \approx \mathbb{P}_{l}$.) We define $J$ using the continuous lattice operations of the topological projective space $\mathscr{P}\left(\mathbb{R}^{2 l+1}\right)$, as follows.

Consider the pencil $\mathscr{R}_{0}$ of all $\mathbb{R}$-lines $R \in P_{1}\left(\mathbb{R}^{2 l+1}\right)$ which contain the origin of the affine space $\mathbb{R}^{2 l} \subseteq P_{0}\left(\mathbb{R}^{2 l+1}\right)$. There is a map $\varphi: \mathscr{R}_{0} \rightarrow \mathscr{L}_{0}$ assigning to every $\mathbb{R}$-line $R$ the unique line $L \in \mathscr{L}$ such that $\bar{L}$ contains $R$. The fact that the incidence relation of the projective space is closed (see 2.1) together with the fact that $\mathscr{L}_{0}$ is compact (see 2.2) implies that $\varphi$ is continuous. Composing $\varphi$ with the continuous join map $\sigma: \bar{A} \rightarrow \mathscr{R}_{0}$ of the projective space, we obtain the desired continuous extension $J=\varphi \circ \sigma$. That $J$ agrees with $j$ on $A$ is easily verified. Moreover, $J$ has the following properties.

2.6 Proposition. Let $\left(\mathbb{R}^{2 l}, \mathscr{L}\right)$ be a topological translation plane, and let $A \subseteq$ $\mathbb{R}^{2 l} \backslash\{0\}$ be an l-dimensional affine subspace that meets each line passing through 0 in at most one point. The projective join map $J=J_{A, 0}$ defined in 2.5 has the following properties.

a) $J: \bar{A} \rightarrow \mathscr{L}_{0} \approx \mathbb{S}_{l}$ is continuous and surjective (remember that $\bar{A} \approx \mathbb{P}_{l}$ ).

b) The map $J$ is an identification, that is, the topology of $\mathscr{L}_{0}$ is the quotient topology with respect to $J$.

c) The set $A \approx \mathbb{R}^{l}$ is mapped homeomorphically onto the set $\mathscr{I} \subseteq \mathscr{L}_{0}$ of lines intersecting $A$.

d) The set $A_{\infty}=\bar{A} \backslash A \approx \mathbb{P}_{l-1}$ is mapped onto the set $\mathscr{D} \subseteq \mathscr{L}_{0}$ of lines disjoint from $A$, and the restriction $J: A_{\infty} \rightarrow \mathscr{D}$ is an identification.

e) The fibers $J^{-1}(L), L \in \mathscr{L}_{0}$, are flats (projective subspaces).

Proof. Continuity of $J$ has been proved. In order to prove surjectivity, consider a line $L \in \mathscr{L}_{0}$ and an arbitrary point $a \in A$. If the intersection $L_{\infty} \cap A_{\infty}$ contains a point $p$, 
then $J(p)=L$. If the intersection is empty, then the $l$-dimensional vector spaces $L$ and $A-a$ are complementary, and this implies that $L \cap A$ is a point $p$. Clearly, $J(p)=L \in \mathscr{I}$, and this proves assertion (a). Conversely, if $L \cap A=\{p\}$, then $A_{\infty} \cap L_{\infty}=\varnothing$; it follows that $J(A) \subseteq \mathscr{I}$ and $J\left(A_{\infty}\right) \subseteq \mathscr{D}$.

Claim (b) follows from the continuity of $J$ together with the facts that $\bar{A} \approx \mathbb{P}_{l}$ is compact and that $\mathscr{L}_{0} \approx \mathbb{S}_{l}$ is a Hausdorff space. By construction, $J$ induces a continuous injective map of the $l$-manifold $A \approx \mathbb{R}^{l}$ into the $l$-manifold $\mathscr{L}_{0} \approx \mathbb{S}_{l}$. The Domain Invariance Theorem, see, e.g., [19], 51.18f, shows that the induced map is a homeomorphism onto its image, and this proves (c). The first part of (d) follows from (a) and (c). The restriction of $J$ is an identification for the same reasons as $J$ itself. Finally, (e) follows from the definition of $J$.

The set $\mathscr{D}$ carries the quotient topology with respect to the restriction $J: A_{\infty} \rightarrow \mathscr{D}$. This topological quotient can also be obtained as follows. Choose an arbitrary point $a \in A$ and map each point $p \in(A-a) \backslash\{0\}$ to the unique line $L \in \mathscr{L}$ containing it. This map is a continuous surjection onto $\mathscr{D}$, and $\mathscr{D}$ carries the quotient topology. The fibers are the sets $((A-a) \cap L) \backslash\{0\}$, where $L \in \mathscr{D}$. Note that the dimension of the fibers is larger by 1 than in the previous description. We prefer, however, the projective point of view, hence we shall not pursue this idea.

2.7 Aims and strategy. We intend to prove a partial negative answer to Problem 1', see Corollary 3.2. The case $l=8$ will remain open, however. This is why we shall give two independent proofs for the main result 3.1. The second one, given in 4.3 , is more direct and does not use the projective join map, whereas the first one, given in Section 3 , yields a comprehensive picture of that map. Conceivably, one of the the two proofs, or both together, will be useful in the future in tackling the last missing case.

Technically, our aim in the first proof is to show that $\mathscr{D}$ has only one element, or in other words, that $A_{\infty}$ contains only one fiber of $J$ (the proof of 3.6 below will show why this is indeed what we want). The method to prove this will be to consider all possibilities for the dimensions of the fibers and to determine the resulting topologies of $\mathscr{D}$, hoping to show that the space $\mathscr{D}$ cannot be the complement of an embedding of $\mathbb{R}^{l}$ into $\mathbb{S}_{l}$, whereas 2.6 says that $\mathscr{L}_{0} \approx \mathbb{S}_{l}$ is the disjoint union of $\mathscr{I} \approx \mathbb{R}^{l}$ and $\mathscr{D}$. This strategy will be successful in all cases except $l=8$.

\section{Nonexistence results}

The core of our nonexistence proof is to establish the following more 'positive' result.

3.1 Theorem. Let $\left(\mathbb{R}^{2 l}, \mathscr{L}\right)$ be a topological translation plane of dimension $2 l=4$ or 8 , and consider an l-dimensional affine subspace $A \subseteq \mathbb{R}^{2 l} \backslash\{0\}$ that intersects every line $L \in \mathscr{L}_{0}$ in at most one point. Then $A$ is a line.

Applying this as indicated in 2.7 we obtain our main result, which partly answers Problem 1': 
3.2 Corollary 1. Weakly topological linear planes other than topological translation planes do not exist except, possibly, in dimension $2 l=16$.

Proof of Corollary 1 . Suppose that $\left(\mathbb{R}^{2 l}, \mathscr{L}\right)$ is a weakly topological linear plane of dimension $2 l \neq 16$. Then 1.4 together with 2.3 shows that the line pencil $\mathscr{L}_{0}$ defines a topological translation plane with line set $\mathscr{L}_{0}+\mathbb{R}^{2 l}$. According to 3.1 , we have $\mathscr{L} \subseteq \mathscr{L}_{0}+\mathbb{R}^{2 l}$. Since each of these two sets defines a join space with point set $\mathbb{R}^{2 l}$, the sets are equal, and the given linear plane is a topological translation plane.

Remember that the definition of a weakly topological linear plane requires the continuity of the join map $j_{p}: q \mapsto q \vee p$ for all points $p$. However, the proof just given does not use the continuity of $j_{p}$ except for one particular point $p=0$. There is nothing special about 0 , and we have the following.

3.3 Corollary 2. For $l \in\{2,4\}$, let $\mathscr{L}$ be a set of l-dimensional affine subspaces of $\mathbb{R}^{2 l}$ such that any two points of $\mathbb{R}^{2 l}$ are on a unique element $L \in \mathscr{L}$. If there is a point $p$ such that the join map $j_{p}$ is continuous, then $\left(\mathbb{R}^{2 l}, \mathscr{L}\right)$ is a topological translation plane.

Before we proceed to the proof of 3.1 , we ask for possible generalizations:

3.4 Problem 2. Describe the class of all translation planes for which Theorem 3.1 is true.

Certainly, 3.1 is not valid in all translation planes; this follows from our examples in 1.12. A case of special interest is, of course, the case of 16-dimensional topological translation planes $(l=8)$. We remark here that in this case the proof of 3.1 fails only when the fibers $X \subseteq A_{\infty}$ of the projective join map $J$ satisfy pdim $X \in\{1,2,3,4,5\}$, with dimension 1 or 2 occurring at least once.

In Section 4, we shall discuss a condition which holds in (locally compact, connected) topological translation planes and implies the assertion of 3.1 for small values of $l$, but not in general.

The Proof of Theorem 3.1 is given as a sequence of lemmas dealing with the various possibilities for the dimensions of the fibers of the projective join map $J=J_{A, 0}$. Another, independent proof will be given in Section 4. We shall always mean the projective dimension pdim $X$ when we speak about the dimension of a flat. This is the same as the topological (manifold) dimension of the point set $P_{0}(X)$. Let $l \in\{2,4,8\}$. It will be tacitly understood that the following lemmas deal with the situation of Theorem 3.1; however, the case $l=8$ will not be excluded.

3.5 Lemma. The projective join map $J$ has at least one nontrivial fiber $X \subseteq A_{\infty}$, that is, a fiber which is not reduced to a point.

Proof. If the assertion is not true, then $J$ is injective, hence $J$ is a homeomorphism by compactness. But $\bar{A} \approx \mathbb{P}_{l}$ and $\mathscr{L}_{0} \approx \mathbb{S}_{l}$ are, not homeomorphic for $l>1$. 
3.6 Lemma. If the projective join map has a fiber $X$ of dimension $\geqslant l-2$, then $A_{\infty}$ is a single fiber, and the assertion of 3.1 is true.

Proof. We have $\operatorname{pdim} X \geqslant \operatorname{pdim} A_{\infty}-1$, hence all other fibers $Y \subseteq A_{\infty}$ are points (remember that fibers must be disjoint). If $X \neq A_{\infty}$, then $J$ is injective on $A_{\infty} \backslash X \approx \mathbb{R}^{l-1}$ and maps this set homeomorphically onto its image because $J$ induces an identification $A_{\infty} \rightarrow \mathscr{D}$. It follows that $\mathscr{D}$ is homeomorphic to the one point compactification $\mathbb{S}_{l-1}$ of $\mathbb{R}^{l-1}$. But then $\mathscr{L}_{0} \backslash \mathscr{D}$ is disconnected by the JordanBrouwer Separation Theorem, see [8], XVII.2.4 or [9], 18.5. This is a contradiction, since we know from $2.6(\mathrm{c})$ and (d) that $\mathscr{I}=\mathscr{L}_{0} \backslash \mathscr{D} \approx \mathbb{R}^{l}$.

Now suppose that $X=A_{\infty}$. This means that $L=J(X)$ is the only line passing through 0 that is disjoint from $\mathrm{A}$, and that the affine subspaces $A$ and $L$ have the same points at infinity. Choose an arbitrary point $a \in A$; then it follows that $A=L+a \in \mathscr{L}$.

3.7 Lemma. The projective join map $J$ has at least one fiber $X \subseteq A_{\infty}$ of $J$ such that $\operatorname{pdim} X \neq \frac{l}{2}-1$.

Proof. Suppose that all fibers are of dimension $\frac{l}{2}-1$. Then the fibers form a spread $\mathscr{S}$ in the projective $(l-1)$-space $A_{\infty}$. Now by its definition, the spread $\mathscr{S}$ is compact in the Grassmann topology on $P_{l / 2-1}\left(A_{\infty}\right)$, hence it gives rise to a topological translation plane on the affine space $A \cong \mathbb{R}^{l}$. Using 2.2 , we infer that $\mathscr{S} \approx \mathbb{S}_{l / 2}$ with respect to the Grassmann topology. On the other hand, arguments like in the proof of 2.6 show easily that the natural map $A_{\infty} \rightarrow \mathscr{S}$ is an identification with respect to the Grassmann topology. Together with $2.6 \mathrm{~d}$ this shows that $\mathscr{D} \approx \mathbb{S}_{l / 2}$, and we can use Lefschetz duality to compute the homology of the complement $\mathscr{L}_{0} \backslash \mathscr{D}$, compare [9], 18.3. The result contradicts the fact that $\mathscr{I}=\mathscr{L}_{0} \backslash \mathscr{D}$ is homeomorphic to $\mathbb{R}^{l}$ according to 2.6 .

3.8 Lemma. The set of points of $A_{\infty}$ forming a 0 -dimensional fiber is open in $A_{\infty}$.

Proof. Remember that a fiber $J^{-1}(L), L \in \mathscr{L}_{0}$, is (the point set of) the intersection $\bar{L} \wedge \bar{A}$. Two $l$-flats in $\mathscr{P}_{2 l}$ cannot be disjoint, hence the stability property (see 2.1 ) implies that the set of all lines $L$ defining a 0 -dimensional fiber is open. By continuity of joining, the corresponding fibers fill an open set of points in $\bar{A}$, and intersecting with $A_{\infty}$ we obtain the assertion.

The next result is purely topological:

3.9 Lemma. Consider a real projective space $\mathbb{P}_{k}, k$ odd, and an identification map $q: \mathbb{P}_{k} \rightarrow X$. If there is a nonempty open subset $U \subseteq \mathbb{P}_{k}$ such that all fibers $q^{-1} q(u)$, $u \in U$, are singletons, then there is a continuous map $X \rightarrow \mathbb{S}_{k}$ that is not homotopic to a constant.

Proof. Choose a topological $k$-disk $D \subseteq U$ and let $Y \approx \mathbb{S}_{k}$ be the quotient space 
obtained from $\mathbb{P}_{k}$ by shrinking the closure of $\mathbb{P}_{k} \backslash D$ to a point. Let $p: \mathbb{P}_{k} \rightarrow Y$ denote the corresponding identification map and $r: \mathbb{S}_{k} \rightarrow \mathbb{P}_{k}$ the universal covering map. There is a continuous map $f: X \rightarrow Y$ such that $p=f \circ q$, and we claim that $f$ is not homotopic to a constant. If this is not true, then also the map $g:=$ $f \circ q \circ r=p \circ r$ is homotopic to a constant. We proceed to show that this is not the case.

The inverse image $r^{-1}(D)$ consists of two disjoint disks that are interchanged by the unique nontrivial deck transformation $\tau: x \mapsto-x$ with respect to $r$. Observe that $\tau$ preserves orientation since $k$ is odd. It follows that both disks are mapped onto $\mathbb{S}_{k}$ with the same orientation, and the mapping degree of $g$ is \pm 2 ; compare [7], Sect. IV.5 or [2], 9.1.

\subsection{Lemma. If $l>1$, then $A_{\infty}$ does not contain a 0 -dimensional fiber.}

Proof. If there is a 0 -dimensional fiber, then 3.8 and 3.9 show that $\mathscr{D} \subseteq \mathscr{L}_{0}$ satisfies the conditions of the Borsuk Separation Theorem [8], XVII.2.1, which asserts that $\mathscr{L}_{0} \backslash \mathscr{D}$ is disconnected; this is a contradiction to $2.6(\mathrm{c})$ and (d), which say that $\mathscr{I}=$ $\mathscr{L}_{0} \backslash \mathscr{D} \approx \mathbb{R}^{l}$.

Note that 3.10 implies the nonexistence of $(l-2)$-dimensional fibers, because fibers are disjoint projective subspaces. In 3.6, we gave an independent and much simpler proof of this fact; that proof may be considered as an illustration of the general argument.

3.11 Summing up the results 3.5 through 3.10, we see that we have proved Theorem 3.1 for $l \in\{2,4\}$. For $l=8$, the open cases are those where all fibers have dimensions $d \in\{1,2,3,4,5\}$ and some number $d \neq 3$ occurs. Since fibers are disjoint, occurrence of $d \in\{4,5\}$ implies occurrence of $d \in\{1,2\}$. For the time being, we cannot prove more.

\section{Bispreads}

R. Riesinger (private communication) raised the question as to whether Theorem 3.1 is valid for all translation planes whose generating spread is also a dual spread, i.e., every vector subspace of codimension one contains exactly one spread element. Such spreads will be called bispreads in the sequel. It is well-known that there are spreads which are not bispreads, see [3], [6], [11], [12]. It is also well-known that every compact spread in a real vector space of finite dimension is a bispread, see [19], 64.10a, hence a positive answer to Riesinger's question would remove the exception $l=8$ from Theorem 3.1 and its corollaries.

It turns out, however, that the answer to Riesinger's question is 'no' in general; we construct counterexamples over every infinite skew field (4.4). Nevertheless, the answer is 'yes' for $l \leqslant 3$, see 4.2. The proof of this result gains even more strength in the topological setting. It can then be used for an alternative proof of Theorem 3.1 (under the original hypotheses). We present this proof, because it might be the key to 
treating the last missing case $l=8$. Yet another problem suggests itself, to which we have no answer:

4.1 Problem 3. Suppose we have a linear plane $(V, \mathscr{L})$ such that each line pencil $\mathscr{L}_{v}$ is a bispread. Is $(V, \mathscr{L})$ necessarily an affine translation plane?

4.2 Theorem. Let $F$ be an arbitrary skew field. Let $\left(F^{2 l}, \mathscr{L}\right)$ be a translation plane of dimension $2 l=4$ or 6 defined by a bispread $\mathscr{L}_{0}$, and consider an l-dimensional affine subspace $A \subseteq F^{2 l} \backslash\{0\}$ that intersects every line $L \in \mathscr{L}_{0}$ in at most one point. Then $A$ is a line.

Proof. Let $\tilde{A}$ be the $(l+1)$-dimensional vector subspace of $F^{2 l}$ spanned by $A$. In general, its intersection with a line $L \in \mathscr{L}_{0}$ is at least one-dimensional, but we can obtain a stronger condition because $\mathscr{L}_{0}$ is a bispread. Indeed, let $H$ be a $(2 l-1)$ dimensional vector subspace of $F^{2 l}$ that contains $\tilde{A}$. Since $\mathscr{L}_{0}$ is also a dual spread, there exists a unique element $L$ of $\mathscr{L}_{0}$ contained in $H$. Then

$$
\operatorname{dim}(L \cap \tilde{A})=\operatorname{dim} \tilde{A}+\operatorname{dim} L-\operatorname{dim}(\tilde{A}+L) \geqslant l+1+l-(2 l-1)=2 .
$$

The same argument shows that $\operatorname{dim} L \cap A_{0} \geqslant 1$, where $A_{0}=A-v, v \in A$, is the direction space of $A$, compare 1.9. It follows that the intersection $L \cap A$ is empty, or else it would contain a coset of $L \cap A_{0}$. Now the direction space $A_{0}$ is the largest vector subspace of $\tilde{A}$ that is disjoint from $A$, hence we have that $L \cap \tilde{A} \subseteq A_{0}$.

It now follows that $L$ is contained in $\tilde{A}$ if and only if $L \subseteq A_{0}$, and dimension considerations show that this is equivalent to $L=A_{0}=A-v$. In this case, $A$ is a coset of $L$ and $A \in \mathscr{L}$.

Now assume that $A$ is not a line. Then $L \nsubseteq \tilde{A}$, and we may choose a different hyperplane $H^{\prime} \geqslant \tilde{A}$, not containing $L$. Then the line $L^{\prime} \in \mathscr{L}_{0}$ contained in $H^{\prime}$ has trivial intersection with $L$, and this carries over to the at least 2-dimensional subspaces $L \cap \tilde{A}$ and $L^{\prime} \cap \tilde{A}$ of $A_{0}$. This is impossible because $\operatorname{dim} A_{0} \leqslant 3$.

Note that we have used the assumption that $l \leqslant 3$ only in the last sentence of the proof. This proof will guide us in the construction of 8-dimensional examples where $A$ is not a line, see 4.4. But first we look at this proof in the topological context; we shall obtain another, independent proof of 3.1 .

4.3 Second proof of 3.1. Recall that every line pencil of a topological translation plane is a bispread. In view of 4.2 , it follows that only the case $l=4$ remains to be considered. We use the notation of the previous proof, except that now $F=\mathbb{R}$. Consider the set $\mathscr{H}$ of all 7-dimensional vector subspaces $H \leqslant \mathbb{R}^{8}$ containing $\tilde{A}$. Intersecting $H$ with some vector space $X \leqslant \mathbb{R}^{8}$ complementary to $\tilde{A}$, we obtain a homeomorphism $\mathscr{H} \approx \mathbb{P}_{2}$.

We assume that $A$ is not a line. In the previous proof, we saw that every $H \in \mathscr{H}$ contains a unique line $L \in \mathscr{L}_{0}$, which intersects $\tilde{A}$ in an at least two-dimensional subspace $\varphi(H)=L \cap \tilde{A}$ of $A_{0}$. We can find $H^{\prime}$ such that $\varphi(H)$ has trivial intersection 
with $\varphi\left(H^{\prime}\right)$; then these are complementary subspaces of the 4-dimensional vector space $A_{0}$. In particular, $\operatorname{dim} \varphi(H)=2$ and $H=L+\tilde{A}$. It now follows that any two distinct hyperplanes $H, H^{\prime} \in \mathscr{H}$ have complementary subspaces $\varphi(H)$ and $\varphi\left(H^{\prime}\right)$ of $A_{0}$ as images. Moreover, $\varphi$ is continuous because incidence is closed. Thus, $\varphi$ is a homeomorphism of $\mathbb{P}_{2}$ onto a partial spread of $A_{0}$. Now in a $2 k$-dimensional real vector space, a partial spread which is a compact $k$-manifold is a spread, see [16], 1.7; moreover, such a spread is homeomorphic to $\mathbb{S}_{k}$, compare 2.2. This is a contradiction.

A large part of this proof works in the case $l=8$, as well. However, we cannot exclude the possibility that $\operatorname{dim} \varphi(H) \in\{2,3,4,5,6\}$ varies depending on $H$, and this makes it impossible to apply the concluding arguments to this situation.

Our final result shows that 3.1 cannot be proved using the bispread condition alone.

4.4 Proposition. Let $F$ be an infinite skew field and consider a vector space $F^{2 l}$, where $l \geqslant 4$. Let $A$ be an l-dimensional affine subspace not containing 0 . Then there is a bispread $\mathscr{L}_{0}$ of l-dimensional subspaces of $F^{2 l}$ such that $A$ intersects every $L \in \mathscr{L}_{0}$ in at most one point but $A$ is not a line of the translation plane defined by $\mathscr{L}_{0}$.

Proof. As in 4.2, we denote the linear span of $A$ by $\tilde{A}$ and the direction space $A-v$, $v \in A$, by $A_{0}$. Like in 1.12, we choose a suitable ordinal $\omega$ with smallest element $1 \in \omega$ and a transfinite enumeration $R_{v}, v \in \omega$, of all one-dimensional vector subspaces of $F^{2 l}$. Similarly, we enumerate the $(2 l-1)$-dimensional subspaces as $H_{v}, v \in \omega$ and the $l$-dimensional subspaces of $F^{2 l}$ as $U_{\mu}, \mu \in \omega$. Inductively, we define $l$-dimensional subspaces $L_{\alpha}$ such that

(1) $L_{\beta} \cap L_{\alpha}=\{0\}$ for $\beta<\alpha$,

(2) $\operatorname{card}\left(L_{\alpha} \cap A\right) \leqslant 1$,

(3) $\operatorname{dim}\left(L_{\alpha} \cap A_{0}\right) \leqslant 2$,

(4) every $R_{v}$ is contained in some $L_{\alpha}$,

(5) every $H_{v}$ contains some $L_{\alpha}$.

This will suffice; note that $A$ is not a line by (3). In each inductive step (assuming that $L_{\beta}$ has been defined for all $\beta<\alpha$ ), we determine first the smallest value of $v$ for which either condition (4) or condition (5) is not yet satisfied and choose $L_{\alpha}$ so that it remedies the defect; if both (4) and (5) fail for the same smallest $v$, preference will be given to (4). This makes it impossible to argue as in 1.12 that always $v(\alpha) \geqslant \alpha$, but still the set $\{v(\alpha) \mid \alpha \in \omega\}$ has the same cardinality as $\omega$, and this implies that conditions (4) and (5) will eventually be satisfied for all $v$. As usual in proofs of this kind, any ambiguity concerning the choice of $L_{\alpha}$ is eliminated by taking the first eligible subspace, i.e. $L_{\alpha}=U_{\mu}$ with minimal $\mu$ satisfying our conditions. The remainder of the proof is concerned with the existence of eligible subspaces.

If condition (4) is the critical one, we define vector subspaces $V_{\beta}=L_{\beta}+R_{v}$ for 
$1 \leqslant \beta<\alpha$ and we set $V_{0}=A_{0}+R_{v}$ if $A_{0}$ does not contain $R_{v}$ while $V_{0}=\tilde{A}$ if $A_{0}$ contains $R_{v}$. Then each $V_{\delta}, 0 \leqslant \delta<\alpha$, has dimension $l+1$. As in 1.12 we obtain an $l$-dimensional vector subspace $U$ that is complementary to each $L_{\beta}$. Moreover, $U \cap V_{0}=R_{v}$ so that either $U$ and $A_{0}$ are complementary or $\operatorname{dim} U \cap \tilde{A} \leqslant 1$, both of which imply (2) for $L_{\alpha}=U$.

Now suppose that $H_{v}$ is the first hyperplane not containing any $L_{\beta}$ with $\beta<\alpha$. Thus, $\operatorname{dim} L_{\beta} \cap H_{v}=l-1$ for $\beta<\alpha$. Furthermore $l \leqslant \operatorname{dim} H_{v} \cap \tilde{A} \leqslant l+1$. Let $k=\operatorname{dim} H_{v} \cap \tilde{A}-(l-1)$ so that $k=1$ or 2 . We have $l \geqslant 4$ and $\operatorname{dim} L_{\beta} \cap A_{0} \leqslant 2$ by (3), hence we may apply Corollary 1.11 to the vector space $H_{v} \cap A_{0}$ and obtain a $k$-dimensional subspace $B$ of $H_{\nu} \cap A_{0}$ such that $B \cap L_{\beta}=\{0\}$ for all $\beta<\alpha$; observe that $A_{0} \subseteq H_{v}$ if $k=2$.

We now define $V_{\beta}=\left(H_{v} \cap L_{\beta}\right)+B$. Then $\operatorname{dim} V_{\beta}=l-1+k=\operatorname{dim} H_{v} \cap \tilde{A}$. By Corollary 1.11 there is a vector subspace $W$ of $H_{v}$ such that $W \cap V_{\beta}=W \cap$ $\left(H_{v} \cap \tilde{A}\right)=\{0\}$ for all $\beta<\alpha$ and $W$ has dimension $2 l-1-(l-1+k)=l-k$. Finally let $U=W+B \subseteq H_{v}$. Then $U$ has dimension $l$ and $U \cap L_{\beta}=\{0\}$ for $\beta<\alpha$ while $U \cap \tilde{A}=B \subseteq A_{0}$. Hence (1), (2) and (3) are satisfied for $L_{\alpha}=U$.

\section{References}

[1] J. André, Über nicht-Desarguessche Ebenen mit transitiver Translationsgruppe. Math. Z. 60 (1954), 156-186. MR 16,64a Zbl 0056.38503

[2] M. A. Armstrong, Basic topology. Springer 1983. MR 84f:55001 Zbl 0514.55001

[3] M. Bernardi, Esistenza di fibrazioni in uno spazio proiettivo infinito. Ist. Lombardo Accad. Sci. Lett. Rend. A 107 (1973), 528-542. MR 49 \#3670 Zbl 0289.50015

[4] M. Biliotti, V. Jha, N. L. Johnson, Foundations of translation planes. Dekker 2001. MR 2002i:51001 Zbl 0987.51002

[5] N. Bourbaki, Elements of mathematics. General topology. Part 2. Hermann, Paris 1966. MR 34 \#5044b Zbl 0301.54002

[6] A. Bruen, J. C. Fisher, Spreads which are not dual spreads. Canad. Math. Bull. 12 (1969), 801-803. MR 41 \#913 Zbl 0186.54303

[7] A. Dold, Lectures on algebraic topology. Springer 1972. MR 54 \#3685 Zbl 0234.55001

[8] J. Dugundji, Topology. Allyn and Bacon Inc., Boston, Mass. 1966. MR 33 \#1824 Zbl 0144.21501

[9] M. J. Greenberg, Lectures on algebraic topology. Benjamin, New York 1967. MR 35 \#6137 Zbl 0169.54403

[10] T. Grundhöfer, R. Löwen, Linear topological geometries. In: Handbook of incidence geometry, 1255-1324, North-Holland 1995. MR 97c:51008 Zbl 0824.51011

[11] H. Havlicek, Dual spreads generated by collineations. Simon Stevin 64 (1990), 339-349. MR 92i:51005 Zbl 0729.51005

[12] H. Havlicek, Spreads of right quadratic skew field extensions. Geom. Dedicata 49 (1994), 239-251. MR 95d:51004 Zbl 0796.51005

[13] N. Knarr, Translation planes, volume 1611 of Lecture Notes in Mathematics. Springer 1995. MR 98e:51019 Zbl 0843.51004

[14] R. Kühne, R. Löwen, Topological projective spaces. Abh. Math. Sem. Univ. Hamburg 62 (1992), 1-9. MR 93g:51013 Zbl 0784.51012

[15] R. Löwen, Compact spreads and compact translation planes over locally compact fields. J. Geom. 36 (1989), 110-116. MR 91c:51024 Zbl 0694.51011 
[16] R. Löwen, Topological pseudo-ovals, elation Laguerre planes, and elation generalized quadrangles. Math. Z. 216 (1994), 347-369. MR 95h:51025 Zbl 0806.51009

[17] H. Lüneburg, Translation planes. Springer 1980. MR 83h:51008 Zbl 0446.51003

[18] D. E. Radford, On the union of closed sets of a finite dimensional vector space. Amer. Math. Monthly 79 (1972), 759-761. MR 46 \#1806 Zbl 0262.54042

[19] H. Salzmann, D. Betten, T. Grundhöfer, H. Hähl, R. Löwen, M. Stroppel, Compact projective planes. de Gruyter 1995. MR 97b:51009 Zbl 0851.51003

Received 13 September, 2002; revised 7 March, 2003

R. Löwen, Institut für Analysis, Technische Universität Braunschweig, Pockelsstraße 14, 38106 Braunschweig, Germany

Email: r.loewen@tu-bs.de

G. F. Steinke, Department of Mathematics, University of Canterbury, Private Bag 4800, Christchurch, New Zealand

Email: g.steinke@math.canterbury.ac.nz

H. Van Maldeghem, Department of Pure Mathematics and Computer Algebra, Ghent University, Galglaan 2, B-9000 Gent, Belgium

Email: hvm@cage.rug.ac.be 